\title{
ON THE TAMAGAWA NUMBER OF QUASI-SPLIT GROUPS
}

\author{
BY K. F. LAI ${ }^{1}$
}

Communicated by H. Rossi, December 1, 1975

1. Introduction. In this paper we give a formula for the Tamagawa number $\tau(G)$ (see [6]) of a connected semisimple quasi-split algebraic group $G$ defined over an algebraic number field $F$. The method used is that of R. P. Langlands (see [2]).

Let $\mathbf{A}$ be the adeles of $F$; $G_{\mathrm{A}}$ the locally compact adele group of $G$ in which the group $G_{F}$ of $F$-rational points is embedded.

Let $B$ be the Borel subgroup of $G$ defined over $F$, and $A$ the maximal torus of $B$ defined over $F . \tau(A)$ is the Tamagawa number of $A . L_{F}$ (resp. $L_{F}^{+}$) denotes the lattice of $F$-rational weights of $G$ (resp. of the simply-connected form of $G$ ). Let $c$ be the index $\left[L_{F}^{+}: L_{F}\right]$. Then the main formula is

THEOREM. $\tau(G)=c \tau(A)$.

2. Sketch of the proof. Let $P$ be the orthogonal projection of $L^{2}\left(G_{F} \backslash G_{\mathrm{A}}\right)$ onto the space of constant functions. Langlands [2] observes the simple relation:

$$
(1,1)\left(P \varphi^{\sim}, P \psi^{\sim}\right)=\left(\varphi^{\sim}, 1\right)\left(1, \psi^{\sim}\right)
$$

where $\varphi^{\sim}, \psi^{\sim} \in L^{2}\left(G_{F} \backslash G_{\mathrm{A}}\right)$ and $(\cdot, \cdot)$ is the inner product on $L^{2}\left(G_{F} \backslash G_{\mathrm{A}}\right)$. As

$$
(1,1)=\int_{G_{F} \backslash G_{\mathrm{A}}} d g
$$

the problem reduces to the computation of the remaining three terms in (1).

Let $G_{\infty}=\Pi_{v \mid \infty} G_{F_{v}}$ where $F_{v}$ is the completion of $F$ at the place $v$ and " $v \mid \infty$ " means that $v$ is infinite. Let $K_{\infty}$ be the maximal compact subgroup of $G_{\infty}$, and $K_{0}=\Pi_{v<\infty} G_{0_{v}}$ where " $v<\infty$ " means that $v$ is finite, $O_{v}$ is the maximal compact subring of $F_{v}$ and $G_{O_{v}}$ is the compact subgroup of $G_{F_{v}}$ consisting of elements with coefficients in $O_{v}$ and whose determinants are units. Put

AMS (MOS) subject classifications (1970). Primary 20G30, 20G35; Secondary 12A70, $12 \mathrm{~A} 80,10 \mathrm{D} 20,32 \mathrm{N10}, 43 \mathrm{~A} 85$.

Key words and phrases. Computation of Tamagawa number, quasi-split algebraic group, Langland's calculation of fundamental domain, $L$-function, torus, Eisenstein series, Weil's conjecture.

1 This paper is based on the author's $\mathrm{Ph}$. D. dissertation, written at Yale University under Professor G. D. Mostow. The problem and the approach were suggested by R. P. Langlands. 
$K=K_{\infty} \cdot K_{0}$. Then there exists a finite set $\left\{g_{i} \in G_{\mathrm{A}} \mid 1 \leqslant i \leqslant n\right\}$ such that

$$
G_{\mathrm{A}}=\bigcup_{i=1}^{n} B_{\mathrm{A}} g_{i} K
$$

Let $N$ be the unipotent radical of $B$, pick continuous functions $\varphi, \psi$ defined on $N_{\mathrm{A}} B_{F} \backslash G_{\mathrm{A}} / K$ such that we have a Fourier integral expression

$$
\varphi(g)=\int_{|\lambda|=\lambda_{0}} \Phi^{\lambda}(g) d \lambda
$$

for a suitable quasi-character $\lambda_{0}$ of $A_{F} \backslash A_{\mathrm{A}}$ and the series

$$
\varphi^{\sim}(g)=\sum_{\gamma \in B_{F} \backslash G_{F}} \varphi(\gamma g)
$$

converges to an element in $L^{2}\left(G_{F} \backslash G_{\mathrm{A}}\right)$. Similarly, we have

$$
\psi(g)=\int_{|\lambda|=\lambda_{0}} \Psi^{\lambda}(g) d \lambda .
$$

The $\Phi, \Psi$ are functions in $\lambda$ and $g$, and there exists a sesquilinear pairing $\langle\cdot, \cdot\rangle$ between these functions such that

$$
(\varphi, 1)=\left\langle\Phi^{\rho}, 1\right\rangle, \quad(1, \psi)=\left\langle 1, \Psi^{\rho}\right\rangle
$$

where $\rho$ is the half sum of the positive roots of $G$.

To evaluate the remaining terms $(P \varphi, P \psi)$, we introduce an unbounded selfadjoint operator $A$ on the closed subspace $L$ of $L^{2}\left(G_{F} \backslash G_{\mathrm{A}}\right)$ generated by the functions $\varphi^{\sim}$ with $\varphi$ of the form indicated above. If $E(x)$ is a right continuous spectral resolution of $A$, then we have

$$
\begin{gathered}
P=E((\rho, \rho))-E((\rho, \rho)-0), \\
\left(P \varphi^{\sim}, P \psi^{\sim}\right)=\frac{1}{c \tau(A)} \lim _{s \rightarrow 1} \frac{\left\langle M\left(w, \rho^{s}\right) \Phi^{\rho^{s}}, \Psi^{\left.w \rho^{-\bar{s}}\right\rangle}\right.}{L(s, A)},
\end{gathered}
$$

where $w$ is the element of the Weyl group that sends every positive root to negative root, $s$ is a complex number, $L(s, A)$ is the $L$-function of $A$ (see [4] , [5]) and $M\left(w, \rho^{s}\right)$ is a linear map on a vector space of functions on $N_{\mathrm{A}} B_{F} \backslash G_{\mathrm{A}} / K$.

There exists a finite set $S$ of places of $F$ such that

$$
M\left(w, \rho^{s}\right) \Phi^{\rho^{s}}(g)=\left(\prod_{v \notin S} \int_{N_{F_{v}}} \Phi^{\rho^{s}}\left(w n_{v}\right) d n_{v}\right)\left(\int_{N_{S}} \Phi^{\rho^{s}}\left(w n_{S} g_{S}\right) d n_{S}\right)
$$

where $g=\left(g_{v}\right) \in G_{\mathbf{A}}$ is such that $g_{v}=1$ if $v \notin S, n_{S} \in N_{S}=\Pi_{v \in S} N_{F_{v}}$.

Let $\bar{N}$ be the unipotent radical of the Borel subgroup opposite to $B$. Write $\bar{N}^{w}=w^{-1} N w \cap \bar{N}$. Then we have

$$
\int_{\bar{N}_{F_{v}}^{w}} \Phi^{\lambda}(\bar{n}) d \bar{n}=\frac{\operatorname{det}\left(I-\left.|\widetilde{\omega}| \sigma \operatorname{Ad} \hat{t}\right|_{\hat{n}} w\right)}{\operatorname{det}\left(I-\left.\sigma \operatorname{Ad} \hat{t}\right|_{\hat{n}} w\right)}
$$


where $\Phi^{\lambda}(1)=1$ (for notation see [3], [4]). Formula (10) is proved first for all rational rank one quasi-split groups by explicit computation and then for the general case by the method of Bhanu-Murti, Gindikin and Karpelevic [1]. From (10) we get

(11) $\lim _{s \rightarrow 1} \prod_{v \notin S} \int_{N_{F_{v}}} \Phi^{\rho^{s}}\left(w n_{v}\right) d n_{v}=\left(\lim _{s \rightarrow 1} \prod_{v \notin S} L_{v}(s, A)\right)\left(\prod_{v \notin S}\right.$ volume $\left.G_{0_{v}}\right)$.

The remaining integral in (9) is calculated by comparing the decomposition of the measure on $G_{\mathrm{A}}$ according to the Iwasawa decomposition and the Bruhat decomposition. We get

$$
\lim _{s \rightarrow 1} \int_{N_{S}} \Phi^{\rho^{s}}\left(w n_{S} g_{S}\right) d n_{S}=\frac{\left\langle\Phi^{\rho}, 1\right\rangle \Pi_{v \in S} L_{v}(1, A)}{\Pi_{v \notin S} \text { volume } G_{0_{v}}} .
$$

The theorem now follows immediately from (1), (2), (6), (8)-(12).

It follows from our theorem that Weil's conjecture on Tamagawa is true for quasi-split group.

\section{RE FERENCES}

1. S. G. Gindikin and F. I. Karpelevič, Plancherel measure for Riemann symmetric spaces of nonpositive curvature, Dokl. Akad. Nauk SSSR 145 (1962), 252-255 = Soviet Math. Dokl. 3 (1962), 962-965. MR 27 \#240.

2. R. P. Langlands, The volume of the fundamental domain for some arithmetical subgroups of Chevalley groups, Proc. Sympos. Pure Math., vol. 9, Amer. Math. Soc., Providence, R. I., 1966, pp. 143-148. MR 35 \#4226.

3. - Problems in the theory of automorphic forms, Lectures in Modern Analysis and Applications, III, Lecture Notes in Math., vol. 170, Springer-Verlag, Berlin, 1970, pp. 18-61. MR 46 \#1758.

4. K. F. Lai, On the Tamagawa number of quasi-split groups, Ph. D. Dissertation, Yale, 1974.

5. T. Ono, Arithmetic of algebraic tori, Ann. of Math. (2) 74 (1961), 101-139. MR 23 \#A1640.

6. - On Tamagawa numbers, Proc. Sympos. Pure Math., vol. 9, Amer. Math. Soc., Providence, R. I., 1966, pp. 122-132. MR 35 \#191.

14B MERRY TERRACE, 4 SEYMOUR ROAD, HONG KONG, HONG KONG 